\title{
Editorial
}

\section{The importance of long axis ventricular function}

Anatomical studies have demonstrated that there are three components to the musculature of the left ventricle, which are arranged longitudinally. These are the papillary muscles, and fibres within the ventricular wall, dominantly subendocardially, and to a lesser extent subepicardially. ${ }^{1}$ The important contribution of longitudinally arranged fibres to overall ventricular function has been recognised for many years. Surgeons now try hard to preserve papillary muscle function in both mitral valve repair and replacement, and patients do better for it. Drugs which improve longitudinal function can produce beneficial effects in patients with ventricular disease. ${ }^{2}$ Both the extent and timing of shortening and thickening of longitudinal fibres is essential to normal systolic function. And yet, despite the ease with which longitudinal ventricular function can be assessed, it has, until relatively recently, been neglected both in the literature and in clinical practice.

In this issue of Heart, Andersson and colleagues describe a double blind trial of metoprolol treatment in patients with ventricular disease, in which they show increases in the amplitude of atrioventricular plane displacement during treatment. ${ }^{3}$ These increases were associated with a reduction in pulmonary capillary wedge pressure and predicted increases in ejection fraction. The association with these haemodynamic improvements was greater than that with changes in short axis performance. These are intriguing findings because the subendocardial fibres are particularly at risk in patients with dilated cardiomyopathy, both from the effects of any large or small coronary disease which may be present, but also from the indirect effects of raised left ventricular diastolic pressures.

Long axis motion of the atrioventricular rings reflects longitudinal ventricular shortening and lengthening, and the extent and timing can conveniently be assessed with $\mathrm{M}$ mode echocardiography. ${ }^{4}$ The velocity of shortening and lengthening can be measured using digitised $M$ modes or tissue Doppler. Because the majority of the longitudinally arranged fibres are located in the subendocardium, long axis function therefore largely reflects subendocardial function. This is therefore useful in assessment of the consequences of ischaemia, to which the subendocardium is particularly sensitive. ${ }^{5}$

Cross sectionally guided $\mathrm{M}$ mode echocardiograms of the left ventricular long axis can be obtained by longitudinal placement of the $M$ mode cursor through the lateral aspect of the mitral annulus and central fibrous body, visualised on the apical four chamber view. Similarly, right ventricular long axis function can be obtained by placing the cursor through the lateral aspect of the tricuspid annulus. Motion of the apex, with respect to the transducer placed at the apex, is insignificant, thus overall lengthening and shortening of the long axis of the left or right ventricles is truly reflected by atrioventricular annular motion.

\section{Pattern of long axis motion}

The normal pattern of long axis motion is characteristic. Shortening of the long axis slightly precedes that of the minor axis, ${ }^{4}$ accounting for the characteristic change in cavity shape during isovolumic contraction. Peak inward motion of the long axis is synchronous with that of the minor axis and aortic closure. The proportional shortening of the long axis is, however, less than that of the short axis so that the ventricle becomes less spherical during contraction. Normal diastolic lengthening of the long axis commences promptly following mitral opening. There is little change in the long axis during diastasis, but atrial contraction pulls the ring up, with further lengthening before subsequent systolic shortening. Thus examination of longitudinal axis $M$ mode echocardiograms can also provide useful information regarding mechanical atrial activity. ${ }^{6}$ Both mitral and tricuspid annular motion may be examined in this way. Systolic descent of the annulus corresponds to systolic venous inflow into the atrium, whether it be right or left. In this way mitral annular motion has been related to pulmonary venous flow, ${ }^{7}$ and tricuspid annular motion to systemic venous inflow down the superior vena cava. ${ }^{89}$ Others have used videotaped two dimensional records for assessment of longitudinal function, ${ }^{10}$ but this lacks the accuracy and temporal resolution of $\mathrm{M}$ mode.

The timing and coordination of events is characteristically disturbed in coronary artery disease with delayed onset of contraction, prolonged shortening beyond aortic closure, and abnormal lengthening during early systole. These effects can be reversed by successful coronary angioplasty. ${ }^{5}$ So characteristic are these abnormalities that identifying them can predict coronary disease in a group of patients undergoing peripheral vascular surgery. ${ }^{11}$ Furthermore, analysis of these abnormalities indicates that disturbances during isovolumic relaxation are in fact the direct consequence of incoordination.

\section{Assessing aortic valve replacement and ventricular disease \\ Long axis function has also been used to assess the effects of} aortic valve replacement upon ventricular function. Abnormalities occur after pulmonary autograft or homograft aortic valve replacement which are indicative of persistent right ventricular longitudinal dysfunction. ${ }^{12}$ Left ventricular longitudinal function appears to recover more quickly, perhaps because of unloading of the hypertrophied ventricle. ${ }^{13}$

In severe ventricular disease there are further characteristic abnormalities. Motion of the mitral annulus is greatly reduced or absent, ${ }^{7}$ and this has been used both in predicting prognosis, ${ }^{14}$ and as a surrogate for ejection fraction. ${ }^{5}$ Typically, not only is the extent of shortening reduced, but the rates of both shortening and lengthening are as well. Andersson's study uses longitudinal function to assess therapeutic effects of a drug and demonstrates that the effects of metoprolol seem greater on the longitudinal fibres than the circumferentially arranged fibres. ${ }^{3}$ These findings are similar to those with intravenous milrinone in dilated cardiomyopathy, suggesting the great sensitivity of the subendocardial fibres to such drugs. ${ }^{2}$ The possibility that there is a greater degree of $\beta$ adrenoceptor down regulation and noradrenaline (norepinephrine) depletion in the subendocardium is an appealing explanation. The changes in long axis function demonstrated by Andersson and colleagues, presumably the direct effect of the drug on longitudinally 
arranged fibres, can now be correlated to reduced wedge pressure and increased ejection fraction and add further weight to the importance of longitudinal fibres in the coordination and integrity of normal ventricular function.

St George's Hospital,

STEPHEN J D BRECKER

Blackshaw Road,

London SW17 0QT, UK

sbrecker@sghms.ac.uk

1 Greenbaum RA, Ho SY, Gibson DG, et al. Left ventricular fibre architecture in man. Br Heart f 1981;45:248-63.

2 Brecker SJD, Xiao HB, Mbaissouroum M, et al. Effects of intravenous milrinone on left ventricular function in ischemic and idiopathic dilated cardiomyopathy. Am 7 Cardiol 1993:71:202-9.

3 Andersson B, Waagstein F, Caidahl K, et al. Early changes in longitudinal performance predict future improvement in global left ventricular function during long term $\beta$ adrenergic blockade. Heart 2000;84:599-605.

4 Jones CJH, Raposo L, Gibson DG. Functional importance of the long axis Jones CJH, Raposo L, Gibson DG. Functional importance of the
dynamics of the human left ventricle. Br Heart 7 1990;63:215-20.

dynamics of the human left ventricle. Br Heart f $1990 ; 63: 215-20$.
5 Henein MY, Priestly K, Davarashvili T, et al. Early changes in left ventricular subendocardial function after successful coronary angioplasty. Br Heart f 1993;69:501-6.
6 Jones CJH, Song GJ, Gibson DG. An echocardiographic assessment of atrial mechanical behaviour. Br Heart 7 1991;65:31-6.

7 Keren G, Sonnenblick EH, LeJemtel TH. Mitral annulus motion. Relation to pulmonary venous and transmitral flows in normal subjects and in patients with dilated cardiomyopathy. Circulation 1988;78:621-9.

8 Hammarström E, Wranne B, Pinto FJ, et al. Tricuspid annular motion. $\mathcal{f} \mathrm{Am}$ Soc Echocardiogr 1991;4:131-9.

9 Wranne B, Pinto FJ, Hammarström E, et al. Abnormal right heart filling after cardiac surgery: time course and mechanisms. $\mathrm{Br}$ Heart $\mathcal{f}$ 1991;66:435-42.

10 Pai RG, Bodenheimer MM, Pai SM, et al. Usefulness of systolic excursion of the mitral anulus as an index of left ventricular systolic function. Am $\mathcal{F}$ Cardiol 1991;67:222-4.

11 Henein MY, Anagnostopoulos C, Das SK, et al. Left ventricular long axis disturbances as predictors for thallium perfusion defects in patients with known peripheral vascular disease. Heart 1998;79;295-300.

12 Carr-White GS, Kon M, Koh T, et al. Right ventricular function after pulmonary autograft replacement of the aortic valve. Circulation 1999; 100(suppl 19):II36-41.

13 Collinson J, Henein M, Flather M, et al. Valve replacement for aortic stenosis in patient with poor left ventricular function: comparison of early changes with stented and stentless valves. Circulation 1999;100(suppl 19):III1-5.

14 Willenheimer R, Cline C, Erhardt L, et al. Left ventricular atrioventricular plane displacement: an echocardiographic technique for rapid assessment of prognosis in heart failure. Heart 1997;78:230-6.

\section{IMAGES IN CARDIOLOGY}

\section{Surgical resection of an asymptomatic aortic valve papillary fibroelastoma in a young man}

An asymptomatic 46 year old man was referred for family screening because of a diagnosis of hypertrophic cardiomyopathy in his father. Cardiovascular examination and the ECG were normal. Transthoracic echocardiogram showed a mobile mass on the non-coronary cusp of the aortic valve and no features of hypertrophic cardiomyopathy. Transoesophageal echocardiography confirmed a discreet $0.5 \mathrm{~cm}$ mobile mass attached to the non-coronary cusp by a stalk and no aortic regurgitation (left image, short axis; right image, long axis). In view of the potential risk of embolism either of the mass or associated thrombus, elective surgical removal was under-

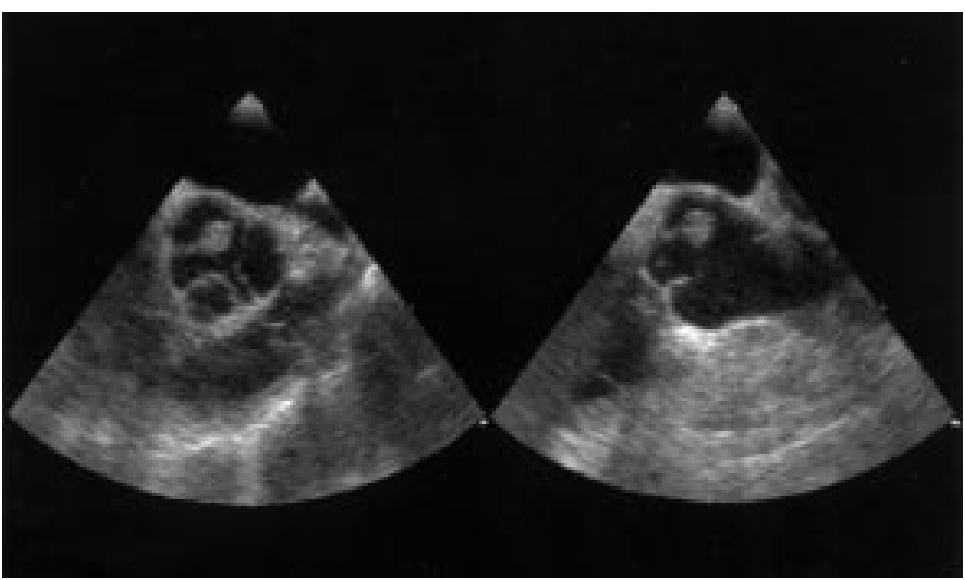

taken. The tumour was shaved off the underside of the leaflet and the base of the lesion coagulated with diathermy, leaving the leaflet intact. The patient made an uneventful recovery and follow-up echocardiography confirmed normal aortic valve function.

Diagnosis of papillary fibroelastoma was suggested by the appearance of macroscopic frondlike structures arising from the tumour when it was emersed in saline. This was confirmed by histological examination.

N R A CLARKE D ROSKELL A P BANNING

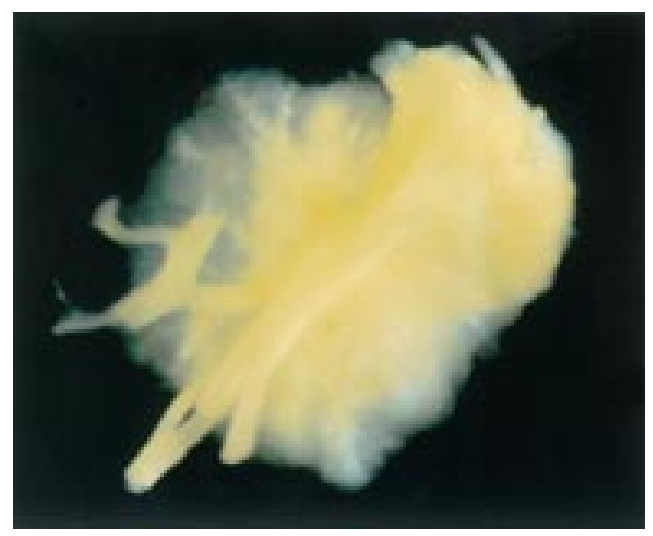

